\title{
Image Quality Determination of a Novel Low Energy X-ray Detector
}

\author{
Hanan Alzahrani, Sion Richards, Iain Sedgwick, Paul Seller, Anastasios Konstantinidis, Gary Royle, Kate Ricketts
}

\begin{abstract}
The demand for adequate image quality with low radiation doses for patients has greatly increased. This is especially true in the case of position verification in radiotherapy which requires a high number of images per patient. This study presents a physical characterisation of a new clinical detector based on a thick layer of structured Thallium activated Cesium Iodide. We made a critical appraisal of its performance for the first time and determined its detective quantum efficiency (DQE) by acquiring the pre-sampling modulation transfer function and normalised noise power spectrum (NNPS). The investigation was conducted with the application of three $x$-ray beam qualities in compliance with the International Electrotechnical Commission (IEC 622201:2003) standard. The spatial resolution and NNPS results led to relatively high $D Q E$ values at all energies: DQEs $(0.5)$ were 0.46 for RQA3, 0.52-0.56 for RQA5 and 0.55-0.59 for RQA7. For CBCT applications, Lassena showed very promising results.
\end{abstract}

\section{INTRODUCTION}

$\mathrm{D}$ igital detectors have gained widespread use in clinical applications. This is due to their high degree of performance and accuracy. The detector under investigation which is referred to as "Lassena" is a wafer scale complementary metaloxide-semiconductor (CMOS) active pixel-based sensor. It is coupled to $1000 \mu \mathrm{m}$ Thallium activated Cesium Iodide (CsI (Tl)) $[1,2]$. The detector consists of two sensors which are stitched together. Each sensor has an active area of $12 \times 14 \mathrm{~cm}^{2}$ with an effective resolution of $2786 \times 2400$ pixels and a pitch of $50 \mu \mathrm{m}$. This detector was designed for general radiographic imaging and cone beam computed tomography (CBCT) applications. These play an essential role in image-guided radiation therapy (IGRT) and adaptive radiotherapy (ART) aspects [3]. Our aim was to critically appraise the detector's performance in order to assess its image quality in terms of physical figures of merit for CBCT applications.

\section{Materials AND Methods}

The characterisation was achieved at University College London (UCL) laboratory with the x-ray source (HS-MP1, Ago $\mathrm{x}$-ray limited, England) having a focal spot of $1 \mathrm{~mm}$, and a tungsten target with aluminium filtration. The measurements were accomplished with three different standard beam conditions: RQA3 (50 kV), RQA5 (70 kV) and RQA7 (90 kV) as RAQ3 was suitable for paediatric extremities imaging, while RQA5 was applicable for adult extremity radiography and RQA7 is commonly employed for CBCT imaging. The test geometry was compliant with International Electrotechnical Commission (IEC 62220-1:2003) standard. The detector was placed at a distance of $150 \mathrm{~cm}$ from the $\mathrm{x}$-ray source. This was to ensure beam uniformity on the detector surface. The half value layer (HVL) had been measured to determine the beam energy required for measurements [4]. The uncertainty was calculated by repeating the measurements three times.

A. Normalised Noise Power Spectrum (NNPS) Determination: The NNPS determines the noise in detector response. 30 dark and 30 bright images (across a range of tube currents $1-8 \mathrm{~mA}$ for $54 \mathrm{kV}, 1-4 \mathrm{~mA}$ for $74 \mathrm{kV}$ and $1-3 \mathrm{~mA}$ for $92 \mathrm{kV}$ ) were acquired below the saturation level. A second-order polynomial fit was applied to correct the beam non-uniformity. The NNPS analysis was conducted according to the IEC protocol by dividing an image into a number of squares referred to as regions of interest (ROIs). Each of these measured $256 \times 256$ pixels with overlapping of 128 pixels. The NPS was acquired as a function of spatial frequency by applying the fast Fourier transform (FFT) [2] using the following equation:

$$
\operatorname{NPS}(\mathrm{u}, \mathrm{v})=\frac{\Delta x \Delta y}{M N_{x} N_{y}} \sum_{m=1}^{M}\left|F F T\left\{I\left(x_{i}, y_{i}\right)-S\left(x_{i}, y_{i}\right)\right\}\right|^{2}
$$

Where $\mathrm{u}$ and $\mathrm{v}$ are the spatial frequencies reflecting $\mathrm{x}$ and $\mathrm{y}, \Delta \mathrm{x}$ and $\Delta \mathrm{y}$ are pixel pitches in $\mathrm{x}$ and $\mathrm{y}$ directions, $\mathrm{Nx}$ and $\mathrm{Ny}$ express the ROI size in $\mathrm{x}$ and $\mathrm{y}$ directions, $\mathrm{M}$ is the ROIs number which is used in averaging and $\mathrm{S}(\mathrm{x}, \mathrm{y})$ and $\mathrm{I}(\mathrm{x}, \mathrm{y})$ are the fitted 2D function and corrected flat field image respectively. The NNPS is obtained by dividing NPS by (large area signal) ${ }^{2}$ which corresponds to the mean digital number (DN) in the image for each dose obtained from STP.

B. Pre-sampling Modulation Transfer Function (pMTF) Determination: The pMTF expresses the spatial resolution in terms of contrast variations in the spatial frequency domain. This was measured by means of the polished edge of tungsten (test device) attached directly to the digital detector approximately along the rows to measure the vertical pMTF by capturing 30 images at the highest current before saturation for each RQA to decrease the statistical noise. Afterwards, the test device was rotated $90^{\circ}$ clockwise with the aim of measuring the horizontal pMTF. The pMTF was obtained in the frequency domain by the modulus of the FFT of the line spread function (LSF) which is the derivative of edge spread function (ESF) extracted from the edge images [2]. The pMTF values were calculated from zero to the Nyquist frequency and the pMTF at zero was normalised to one.

C. Detective Quantum Efficiency (DQE) Measurement: The $\mathrm{DQE}$ determines the ability of an imaging system to transfer the input $\mathrm{x}$-ray signal to an image. It was computed using the following equation:

$$
\operatorname{DQE}(\mathrm{f})=\left(\frac{S N R_{\text {Out }}}{S N R_{I n}}\right)^{2}=\frac{p M T F^{2}(f)}{\frac{\Phi}{K_{a}} * \mathrm{~K}_{a} * N N P S(f)}
$$

Where SNRout is the signal-to-noise ratio of the output signal on the image. It can be measured from the acquired images by
Manuscript received December 13, 2018

Hanan Alzahrani is with University College London, London, WC1E 6BT, UK (e-mail: hanan.alzahrani.13@ucl.ac.uk).

Sion Richards, Iain Sedgwick and Paul Seller are with Rutherford Appleton Laboratory, Didcot, OX11 0QX, UK.
Anastasios Konstantinidis is with The Christie NHS Foundation Trust, Manchester M20 4BX, UK.

Gary Royle and Kate Ricketts are with University College London, London, WC1E 6BT, UK. 
dividing the $\mathrm{pMTF}^{2}$ by the averaged NNPS of the digital $\mathrm{x}$-ray imaging device. The SNRIn is the signal to noise ratio of the input signal to the detector which can be estimated by multiplying the photon fluence per exposure ratio $\left(\Phi / K_{\mathrm{a}}:\right.$ in $\mathrm{x}$-ray per $\mathrm{mm} 2 / \mu \mathrm{Gy}$ ) by air-Kerma (Ka: in $\mu \mathrm{Gy})$ where $(\Phi)$ was provided by IEC $62220-1$. It was assumed that the detector behaved as ideal photon counter [4-7].

\section{RESULTS AND DISCUSSION}

A. Normalised Noise Power Spectrum (NNPS): The NNPS at different energies states that the noise decreased as the radiation energy and dose increased (Fig.1). This reduction is due to the intensification of the signal resulting from a high number of photons interacting with the scintillator. Therefore, the absorption efficiency increases. This finding implies that NNPS is heavily dependent on exposure. Lassena was compared to other available CBCTs on the market. The results are illustrated in table 1 [8-12]. It is apparent that Lassena has the lowest noise due to the thick scintillator.

B. Pre-sampling Modulation Transfer Function ( $p M T F)$ : Generally, the resolution of the imaging system degraded as a function of frequency. It was also found that the pMTFs are independent of the beam quality within the investigated range. However, increasing the radiation energy slightly improved the resolution at low frequencies between 1 and 3 line pairs per millimetre $(\mathrm{lp} / \mathrm{mm})$ due to higher absorption depth which limited the spread of the scintillation photons at generated by the higher energy x-rays. The pMTF reaches $50 \%$ at $0.9,1.08$ and $1.1 \mathrm{lp} / \mathrm{mm}$ for 54,74 and $92 \mathrm{kV}$ beam qualities respectively (Fig.2). It is known that the frequency corresponding to $10 \%$ MTF describes the limiting resolution of a system and it is around $3 \mathrm{lp} / \mathrm{mm}$ for all three beam qualities. In general radiology, the adequate limiting resolution for a detector ranges between 3 and $5 \mathrm{lp} / \mathrm{mm}$ [13]. As a result, the detector under investigation has a sufficient imaging resolution. Looking at table 1 for detectors comparison, it is particularly notable that Lassena has the highest limiting resolution which is $3 \mathrm{lp} / \mathrm{mm}$ due to the small pixel size.

C. Detective Quantum Efficiency (DQE): Ideally, detectors should have DQE of unity at all spatial frequencies. However, in reality, the DQEs of three radiation energies declined as a function of frequency. This is the case for all radiation detectors. On the other hand, DQEs escalated when the radiation dose and energy rose but at high frequencies the DQE became less exposure dependent as illustrated in Fig. 3. The DQE (0.5) values are around 0.46 for all doses for RQA3 and they range from $0.52-0.56$ for RQA5. Finally, the values of DQE (0.5) for RQA7 are approximately 0.55-0.59.

\begin{tabular}{|c|c|c|c|c|}
\hline \multicolumn{5}{|c|}{$\begin{array}{l}\text { TABLE I. COMPARISON BETWEEN THE LASSENA AND OTHER AVAILAB } \\
\text { DETECTORS THAT CAN BE USED FOR CBCT IN THE RADIOTHERAPY } \\
\text { DEPARTMENTS [8-12]. }\end{array}$} \\
\hline & Varian & Elekta & Siemens & Lassena \\
\hline ical size $\left(\mathrm{cm}^{2}\right)$ & $39.73 \times 29.8$ & $41 \times 41$ & $41 \times 41$ & $24 \times 14.4$ \\
\hline lage depth(bit) & 16 & 16 & $16 / 12$ & 14 \\
\hline ixel pitch $(\mathrm{cm})$ & 0.038 & 0.05 & 0.04 & 0.005 \\
\hline Max frame rate(fps) & 30 & 5.5 & 25 & 30 \\
\hline ITF $50 \% / 10 \%(\mathrm{lp} / \mathrm{mm})$ & $0.54 / 0.93$ & $0.28 / 0.45$ & $0.3 / 0.5$ & $1.5 / 3$ \\
\hline mage noise $(\%)$ & 0.7 & 1.4 & 2.7 & 0.02 \\
\hline
\end{tabular}

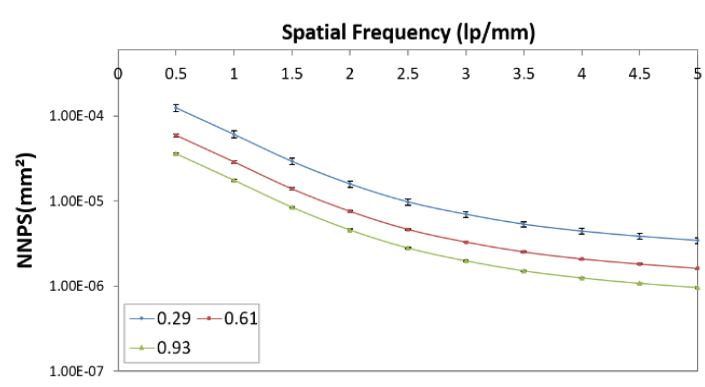

Fig. 1. An example of 1D NNPS pattern at $92 \mathrm{kV}$ for different $K_{a}$ values in $\mu \mathrm{Gy}$.

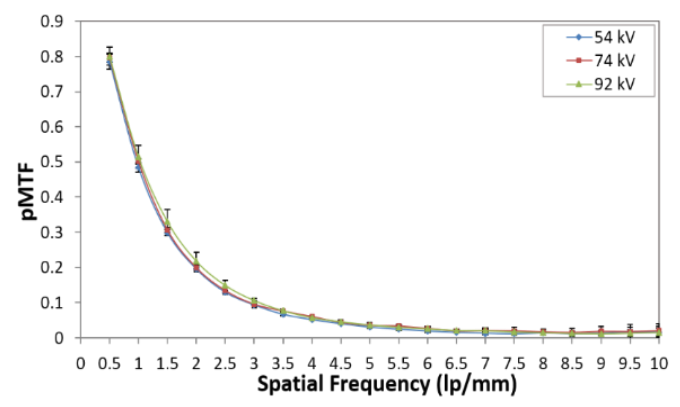

Fig. 2. The averaged pMTFs for Lassena at three different energies.

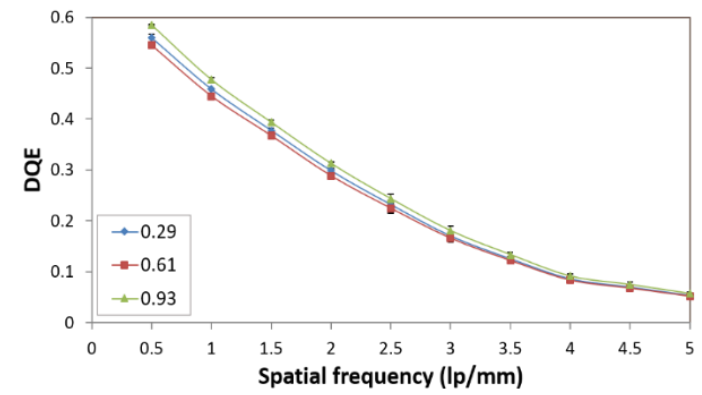

Fig. 3. An example of DQE pattern for different $K_{a}$ values $(\mu G y)$ at $92 \mathrm{kV}$.

\section{CONCLUSION}

The main aim of this collaborative project was to achieve a characterisation of the detector performance for the first time over a range of radiation energies and currents to determine its image quality. The DQEs were quantified at three radiation beam qualities recommended by the IEC standard. The spatial resolution and NNPS results led to relatively high DQE values at all energies: DQEs (0.5) were 0.46 for RQA3, $0.52-0.56$ for RQA5 and 0.55-0.59 for RQA7. For CBCT applications, Lassena showed very promising results.

\section{REFERENCES}

Michail, C. et al., 2015. Experimental measurement of a high-resolution CMOS detector coupled to CsI scintillators under X-ray radiation. Radiation Measurements, 74, pp.39-46.

Konstantinidis, A.C. et al., 2013. X-ray performance evaluation of the dexela cmos aps x-ray detector using 60(5), pp.3969-3980.

Sedgwick, L, LASSENA: A 6.7 Megapixel, 3-sides Buttable Wafer-Scale CMOS Sensor using a novel gridaddressing architecture.

IEC, 2003. Medical electrical equipment - Characteristics of digital X-ray imaging devices - Part 1: Determination

of the detective quantum efficiency.
Jones, D.P. (Deric P., 2010. Biomedical sensors, Momentum Press.

Illers, H., Vandenbroucke, D. \& Buhr, E., 2004. Measurement of correlated noise in images of computed radiography systems and its influence on the detective quantum efficiency. in Medical Imaging 2004: Physics of Medical Imaging, Martin J. Yaffe, Michael J. Flynn, Editors, Proceedings of SPIE Vol. 5368, 639-647 (2004), 5368, pp.639-647. Beutel, J., 2000. Handbook of medical imaging, SPIE Press

Lechuga, L. \& Weidlich, G.A., 2016. Cone Beam CT vs. Fan Beam CT: A Comparison of Image Quality and Dose Delivered Between Two Differing CT Imaging Modalities. Cureus, 8(9).

Srinivasan, K. et al., 2014. Applications of linac-mounted kilovoltage Cone-beam Computed Tomography in modern radiation patient dose. Journal of medical physics, $36(4)$, pp.205-12.

Elstrøm, U. V. et al., 2011. Evaluation of image quality for different $\mathrm{kV}$ cone-beam $\mathrm{CT}$ acquisition and reconstruction methods in the head and neck region. Acta Oncologica, 50(6), pp.908-917.

Chan, M.F. et al., 2011. Evaluation of imaging performance of major image guidance systems. Biomedical Imaging and Intervention Journal, 7(2).

Bushberg, J.T. et al., 1994. The essential physics of medical imaging, LWW. 